\title{
Triple-Phase Spiral CT
}

National Cancer Institute

\section{Source}

National Cancer Institute. Triple-Phase Spiral CT. NCI Thesaurus. Code C19907.

A form of computed tomography (CT) in which images are taken during three distinct timing points (phases) following the injection of contrast material: arterial, venous, and delayed. This form of CT imaging is most commonly used to image hepatic lesions. 